\title{
What is the Parasitic nematode Anisakis doing in host fish?
}

\author{
SATOSHI SUGAYA ${ }^{1}$, YUKO TAKIZAWA ${ }^{2}$ \\ AND YOSHITO CHIKARAISHI ${ }^{2,3}$ \\ ${ }^{2}$ Institute of Low Temperature Science, Hokkaido University \\ ${ }^{3}$ Japan Agency for Marine-Earth Science and Technology
}

${ }^{1}$ Fisheries Sciences, Hokkaido University

(ychikaraishi@lowtem.hokudai.ac.jp)

The parasitic nematode Anisakis is one of major parasites frequently found in fish, squid, and marine mammals including dolphins and whales, and cases severe abdominal pain if human eats infected fish or squid. However, little knowledge is available in what the parasites are doing in the host fish species and how they can survive and remain infective in their unique life cycles. In the present study, to enhance our knowledge on the parasite, we investigated trophic level (TL) and potential diet resources $\left(\delta^{15} \mathrm{~N}_{\text {Resource }}\right)$ based on stable nitrogen isotopic composition $\left(\delta^{15} \mathrm{~N}\right)$ of glutamic acid and phenylalanine in Anisakis larvae and their host fish Pleurogrammus azonus.

The TL of Anisakis larvae (2.6-2.9) is considerably smaller than that of muscle in the host fish (3.6). Moreover, the $\delta^{15} \mathrm{~N}_{\text {Resource }}$ values largely vary among individual Anisakis larvae specimens $\left(2.4 \%{ }^{-4.7 \% o}\right)$, and several specimens are clearly smaller than the value of muscle in the host fish (4.3\%). These results reveal that Anisakis larvae have never fed on the host fish tissues/organs even though they are found in the fish, and suggest that Anisakis larvae can survive as a dormant phase, remain infective, and be accumulated in the paratenic host fish for the effective infection to the definitive hosts.

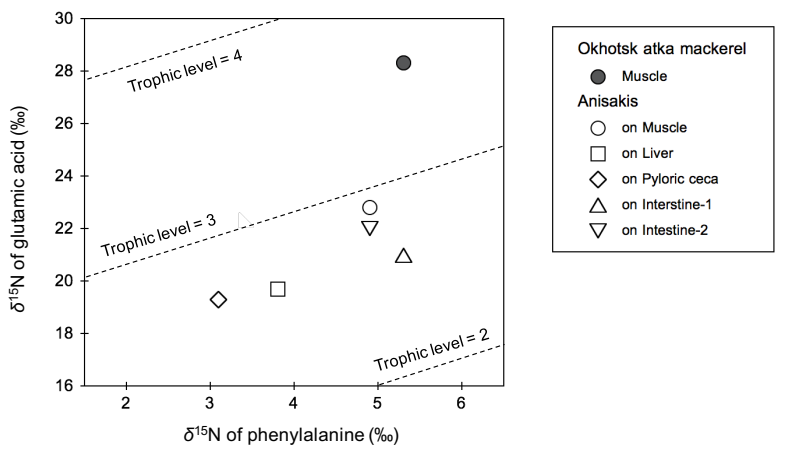

Fig 1. Cross-plot of $\delta^{15} \mathrm{~N}$ values between glutamic acid and phenylalanine for Anisakis larvae (open symbol) and the host fish Pleurogrammus azonus (filled symbol). 